\title{
Burden and clinical characteristics of high grade tuberculosis destroyed lung: a nationwide study
}

\author{
Deokjae Han ${ }^{1}$, Hwa Young Lee ${ }^{1}$, Kyeongju Kim ${ }^{1}$, Taehoon $\mathrm{Kim}^{2}$, Yeon-Mok $\mathrm{Oh}^{3}$, Chin Kook Rhee ${ }^{1}$ \\ ${ }^{1}$ Division of Pulmonary and Critical Care Medicine, Department of Internal Medicine, The Catholic University of Korea, Seoul, Korea; ${ }^{2}$ Division \\ of Pulmonary and Critical Care Medicine, Department of Internal Medicine, CHA Bundang Medical Center, CHA University, Seongnam-si, \\ Gyeonggi-do, Korea; ${ }^{3}$ Department of Pulmonary and Critical Care Medicine, Clinical Research Center for Chronic Obstructive Airway Diseases, \\ Asan Medical Center, University of Ulsan College of Medicine, Seoul, Korea \\ Contributions: (I) Conception and design: CK Rhee; (II) Administrative support: YM Oh, CK Rhee, K Kim; (III) Provision of study material or \\ patients: CK Rhee, D Han, HY Lee, T Kim; (IV) Collection and assembly of data: CK Rhee, K Kim; (V) Data analysis and interpretation: CK Rhee, \\ K Kim, D Han, HY Lee; (VI) Manuscript writing: All authors; (VII) Final approval of manuscript: All authors. \\ Correspondence to: Yeon-Mok Oh, MD, PhD; Chin Kook Rhee, MD, PhD. Department of Pulmonary and Critical Care Medicine, Clinical Research \\ Center for Chronic Obstructive Airway Diseases, Asan Medical Center, University of Ulsan College of Medicine, 88, Olympic-ro 43-gil, Songpa- \\ gu, Seoul, 05505, South Korea; Division of Pulmonary and Critical Care Medicine, Department of Internal Medicine, The Catholic University of \\ Korea, 222, Banpodae-ro, Seocho-gu, Seoul 06591, South Korea. Email: ymoh55@amc.seoul.kr; chinkook77@gmail.com.
}

Background: Little clinical information on high grade tuberculosis destroyed lung (TDL) is available. The aim of this study was to investigate the characteristics and healthcare utilization of high grade TDL patients, and compared the differences between acute exacerbation and healthcare burden according to inhaler compliance.

Methods: This was an observational retrospective cohort study using the Korean Health Insurance Review and Assessment (HIRA) service database (2011-2015). Patients diagnosed with high grade TDL in 2011 were enrolled and reviewed for 5 years. The patients were further divided into adherent and non-adherent groups. Their socioeconomic outcomes according to treatment adherence in 2012 were analyzed.

Results: Among the 13,346 patients diagnosed with high grade TDL, 3,637 were assigned to the adherent group and 9,709 to the non-adherent group. Overall, 65.91\% of the enrolled patients were male and the mean age of the study population was $64.68 \pm 10.06$ years. All patients visited a tertiary hospital, but $99.04 \%$ and $69.74 \%$ also visited primary and secondary hospitals, respectively. The mean number of hospital admissions per year was $1.38 \pm 2.03$ times per patient. The average total annual per-patient cost was US\$4,140.95 $\pm 3,715.01$ and each patient spent a total of $56.21 \pm 45.28$ days per year using hospital services. The majority of the patients in the adherent group were male $(80.09 \%$ vs. $60.60 \%, \mathrm{P}<0.01)$, and were of older age $(65.71 \pm 9.35$ vs. $64.29 \pm 10.28, \mathrm{P}<0.01)$ than the non-adherent group. The frequencies of visiting a tertiary hospital ( 96.87 vs. $90.12 \%, \mathrm{P}<0.01$ ), the total mean healthcare utilization costs (US $\$ 4,151.77 \pm 4,084.76$ vs. US\$3,592.54 $4,229.93, \mathrm{P}<0.01)$, and the frequencies of exacerbations $(0.72 \pm 2.03$ vs. $0.46 \pm 1.51, \mathrm{P}<0.01)$ were higher in the adherent group. However, healthcare services were used on significantly fewer days in the adherent group $(52.96 \pm 50.87$ vs. $56.67 \pm 50.81, \mathrm{P}<0.01)$.

Conclusions: High grade TDL imposes a high socioeconomic burden in Korea. Estimated medical costs and exacerbation event rate were higher in the adherent group whereas number of days of healthcare usage was significantly lower.

Keywords: Chronic obstructive lung disease (COPD); adherence; tuberculosis destroyed lung (TDL)

Submitted Mar 10, 2019. Accepted for publication Sep 23, 2019.

doi: $10.21037 /$ jtd.2019.09.63

View this article at: http://dx.doi.org/10.21037/jtd.2019.09.63

(c) Journal of Thoracic Disease. All rights reserved. 


\section{Introduction}

The worldwide incidence of tuberculosis (TB) in 2016 was 10.4 million and the number of deaths from TB in that same year was 1.7 million, according to the WHO Global Tuberculosis Report 2017 (1). Tuberculosis destroyed lung (TDL), in which the lung parenchyma is extensively damaged, is one of the most devastating sequelae of TB. The destructive effect of TB on the lung causes bronchiectasis and airway flow limitations, and thus progressive lung function decline, frequent exacerbations, respiratory failure and pulmonary disabilities (2-6). Several studies have shown that TDL is one of the most important risk factors for chronic obstructive lung disease (COPD) (2,7-15). Given the clinical similarities between TDL and COPD, the symptoms of both are commonly treated with various types of bronchodilators. The few studies that have specifically addressed the effect of inhaled bronchodilators on TDL noted significantly improved treatment outcomes with inhaled tiotropium and indacaterol $(16,17)$.

No formal treatment guidelines for TDL because little is known about its clinical characteristics or burden in affected patients. Evaluations of treatment efficacy based on clinical and socioeconomic factors are also lacking. There are also no standard disease classification criteria that take the severity of TDL into account. Although it may be difficult to identify the severity with claim data because there is no pulmonary function data, our previous study defined severe patient as 'high grade' by using history of exacerbation and drug use (18). We intended to evaluate the burden of severe TDL patient by using the high-grade definition developed previously (18).

Korea has a unique medical insurance system, managed and supervised by the Korean government, called the Korean National Health Insurance (KNHI) system. All Koreans are compulsorily registered within the KNHI, and all healthcare utilization and essential medical data are managed by the Korean Health Insurance Review and Assessment (HIRA) service. The epidemiological data of the $\mathrm{KNHI}$ system can therefore be used to conduct nationwide studies.

This observational retrospective cohort study was performed using the HIRA database, specifically, data pertaining to the subset of patients with high grade TDL. This group of patients was expected to have higher medical service utilization costs than patients with other diseases. Within the group of patients with high grade TDL, differences in treatment adherence were expected. The aim of this study was to investigate the characteristics, drug use, and health care utilization of high grade TDL patients, and compare the differences between acute exacerbation, health care utilization and cost according to inhaler compliance.

\section{Methods}

\section{Study population and design}

\section{Basic database}

The HIRA claims dataset and diagnostic criteria of the International Codes of Disease, $10^{\text {th }}$ edition, Clinical Modification (ICD-10-CM), were used in this study. The HIRA database, which contains official healthcare service information for nationwide population, was searched to identify patients diagnosed with TDL (ICD code B90 or J984) between January 1, 2011 and December 31, 2011. Patients who had both ICD codes as the primary diagnosis, or as the second to fifth sub-diagnosis and were older than 40 years of age were included in the study. Data on patient age, sex, insurance type, comorbidities, drug prescriptions (drug name and rate of prescription), billing codes and health care utilization status were extracted from the database. Information on drug prescriptions and healthcare visits was based on 5-year (2011-2015) claims data and was retrospectively analyzed. Medical costs and days of healthcare utilization were analyzed separately according to use of outpatient and inpatient services and were counted only for services related to TDL. All costs were assessed in US dollars (US\$), based on an exchange rate with the Korean Won (KRW) on September 5, 2018 of US\$ $1=1,117.60 \mathrm{KRW}$.

\section{High grade TDL}

Among the TDL patients enrolled in the database, those who fulfilled both of the following criteria were defined as have high grade TDL: (I) visited a tertiary hospital at least once between January 1, 2011 and December, 31, 2011 and (II) prescribed one or more of the following during the stated period: (i) at least two packages each of both inhaled corticosteroid/long-acting $\beta 2$ agonist combination inhalers (ICS/LABA) and long-acting muscarinic antagonist inhalers (LAMA); (ii) systemic steroids, i.e., intravenous corticosteroids (IVCS) and oral corticosteroids (OCS), at least twice, and at least two packages of LAMAs; (iii) both IVCSs and OCSs, at least twice, and at least two packages of ICS/LABAs. Patients with high grade TDL were evaluated for 5 years, between January 1, 2011 and December 31, 
2015, to assess their disease burden.

\section{Groups}

Patients defined as having high grade TDL based on claims data in 2011 were divided into two subgroups to compare outcomes according to treatment compliance during a 1 -year period (2012). The adherent group comprised patients prescribed (I) 10 or more packs of ICS/LABAs or (II) 10 or more packs of LAMAs during the stated period. The non-adherent group included all other patients with high grade TDL not included in the adherent group. For both groups, claims data in 2012 were available and allowed comparison of patient outcomes for the period between January 1, 2012 and December 31, 2012.

Disease exacerbation was defined as an unexpected or unscheduled visit to a hospital emergency department, or hospitalization due to worsening of respiratory symptoms. Patients with moderate exacerbations were those (I) with outpatient service billing codes that included ICD code B90 or J948 and (II) who were prescribed systemic steroids, including IVCSs and OCSs. Severe exacerbation described patients (I) whose inpatient service or emergency room (ER) billing codes included ICD code B90 or J948, and (II) who were prescribed systemic steroids.

\section{Statistical analysis}

All statistical analyses were performed using SAS software (ver. 9.2; SAS Institute, Inc., Cary, NC, USA). The results are expressed as means \pm standard deviation for continuous variables and as proportions for categorical variables. Differences between groups were assessed using Student's $\mathrm{t}$-test for continuous variables and the $\chi^{2}$ test for categorical variables. Negative binomial regression was applied to analyze the effect of adherence on disease exacerbation, and multiple linear regression was used to identify the factors affecting medical costs and days of healthcare utilization. All tests were two sided, and a $\mathrm{P}$ value $<0.05$ indicated statistical significance.

\section{Ethics statement}

To protect their confidentiality, all patients were assigned and anonymous identification code in the HIRA database that prevented the authors from identifying them. The study was approved by the Institutional Review Board of Seoul St. Mary's Hospital, which waived the requirement for informed consent.

\section{Results}

\section{Study population}

Six hundred twenty thousand and six hundred sixty-nine patients diagnosed with TDL between January 1, 2011 and December 31, 2011. After excluding deceased patients and those who never visited a tertiary hospital, 13,346 patients diagnosed with TDL in 2011 fulfilled the criteria for high grade TDL and could be reviewed for 5 years (January 1, 2011 to December 31,2015) to analyze the burden of TDL. Among high grade TDL patients, 3,637 could be assigned to the adherent group and 9,709 to the non-adherent group. Both groups were reviewed during 2012.

\section{Burden of bigh grade TDL}

Baseline characteristics of patient with high grade TDL The baseline characteristics of the patients with high grade TDL in 2012 are shown in Table 1. Among the 13,346 patients, 8,797 (65.91\%) were male. The mean age was $64.68 \pm 10.06$ years and most patients were in their 60 s $(35.87 \%)$. Primary hospitals being visited more frequently than secondary hospitals $(99.04 \%$ vs. $69.74 \%)$. The most common, but not the only, comorbidities were gastroesophageal reflux disease (GERD, 87.07\%), hyperlipidemia (77.07\%) and hypertension (68.85\%).

The baseline characteristics of the adherent and nonadherent groups (2012) are also shown in Table 1. A higher percentage of patients were male in the adherent than in the non-adherent group $(80.09 \%$ vs. $60.60 \%, \mathrm{P}<0.01)$ and the mean age was higher $(65.71 \pm 9.35$ vs. $64.29 \pm 10.28$, $\mathrm{P}<0.01)$. Patients in the adherent group were more likely than those in the non-adherent group to have utilized a tertiary hospital $(96.87 \%$ vs. $90.12 \%, \mathrm{P}<0.01)$ rather than a primary $(91.34 \%$ vs. $95.06 \%, \mathrm{P}<0.01)$ or secondary $(31.95 \%$ vs. $40.88 \%, \mathrm{P}<0.01)$ hospital.

The five most prevalent comorbidities were the same in both groups; hypertension, diabetes mellitus, hyperlipidemia, GERD and osteoporosis. The difference in prevalence between the adherent and non-adherent groups was significant for hyperlipidemia $(37.53 \% \mathrm{vs}$. 43.14\%, $\mathrm{P}<0.01)$, GERD (47.2\% vs. $52.8 \%, \mathrm{P}<0.01)$ and osteoporosis ( $16.52 \%$ vs. $20.98 \%, \mathrm{P}<0.01)$.

\section{Healthcare utilization}

Table 2 shows the healthcare utilization frequency of patients with high grade TDL. Both the number of days of healthcare utilization during the study period and the 
Table 1 Baseline characteristics of the patients with high grade TDL

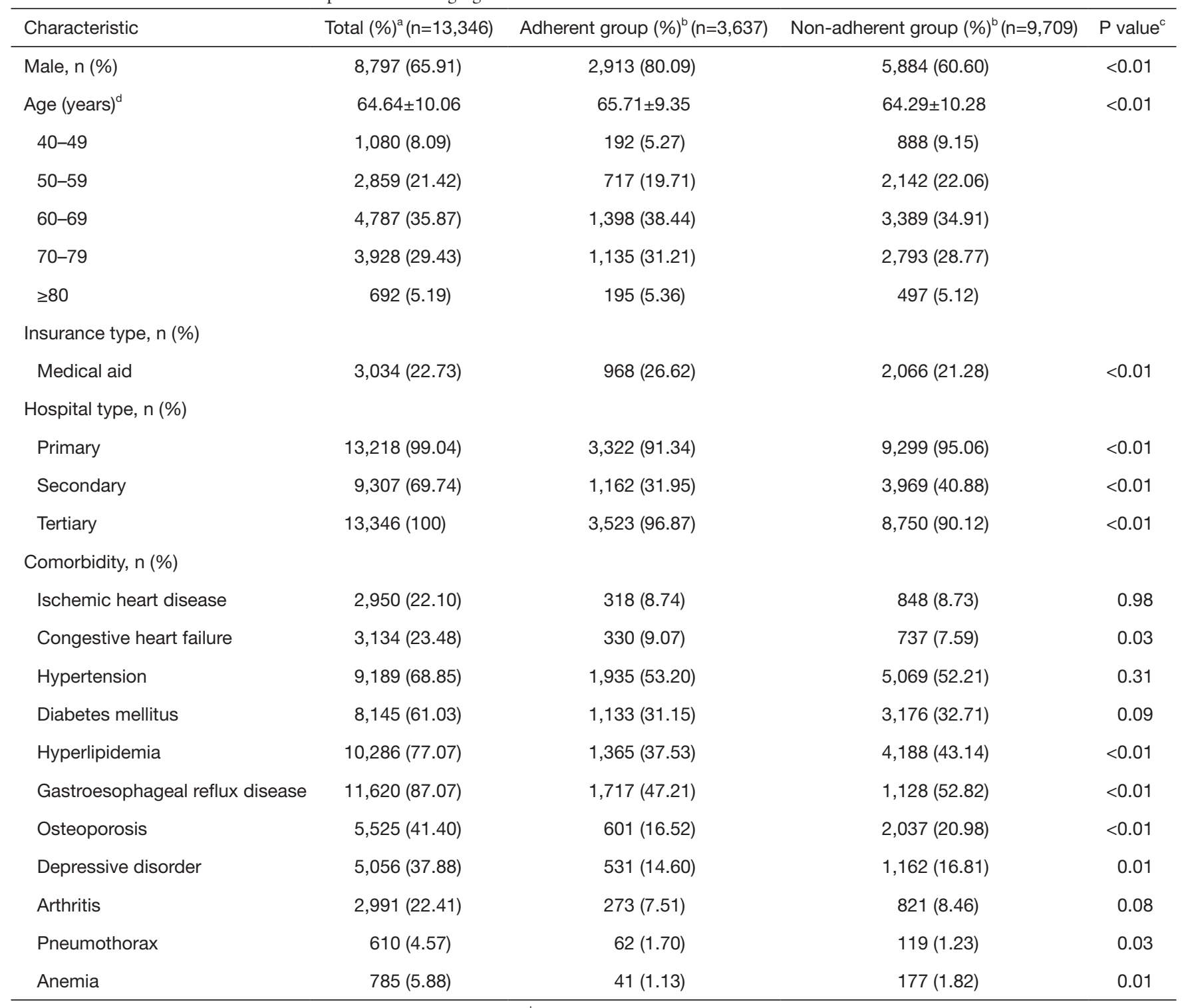

${ }^{a}$, the entire group of high grade TDL patients (2011-2015)); ${ }^{b}$, groups divided by treatment compliance (2012); ${ }^{c}$, for adherent group and non-adherent group; ${ }^{d}$, mean \pm SD. TDL, tuberculosis destroyed lung; SD, standard deviation.

annual average frequency of utilization were determined for each patient. An ER visit or hospitalization on a general ward $(\mathrm{GW})$ at least once was documented for 11,844 $(88.75 \%)$ patients, with an annual average of $1.38 \pm 2.03$ visits per patient. Intensive care unit (ICU) admission was required by 2,243 (16.81\%) patients, with an annual average of $0.32 \pm 0.29$ visits per patient.

Table 2 also shows the frequencies of the various examinations of TDL disease activity. Pulmonary function tests (PFTs, 95.14\%) were the most frequently performed, followed by chest X-ray (67.56\%) and chest computed tomography (CT, $8.51 \%)$. The annual per-patient frequency of PFT, chest X-ray and chest CT was $1.14 \pm 1.09,0.88 \pm 1.0$ and $0.38 \pm 0.34$, respectively.

The healthcare service utilization frequency did not differ significantly between the two groups (2012), nor did the percentage of patients who visited the ER or were hospitalized on a GW at least once (45.89\% vs. $46.74 \%$, $\mathrm{P}=0.38)$. The group difference in annual average per-patient frequency of ER visits or GW hospitalization was also no 
Table 2 Healthcare utilization by patients with high grade TDL

\begin{tabular}{|c|c|c|c|c|c|c|c|c|}
\hline $\begin{array}{l}\text { TDL-related healthcare } \\
\text { utilization }\end{array}$ & \multicolumn{4}{|c|}{$\mathrm{N}(\%)$} & \multicolumn{4}{|c|}{ Utilization frequency ${ }^{d}$, mean $\pm S D$} \\
\hline ER visit or GW hospitalization & $11,844(88.75)$ & $1,699(45.89)$ & $4,538(46.74)$ & 0.38 & $1.38 \pm 2.03$ & $1.16 \pm 2.24$ & $1.23 \pm 2.3$ & 0.11 \\
\hline ICU care & $2,243(16.81)$ & $138(3.79)$ & 327 (3.37) & 0.23 & $0.32 \pm 0.29$ & $0.05 \pm 0.27$ & $0.04 \pm 0.23$ & 0.13 \\
\hline Chest X-ray & $9,017(67.56)$ & $979(26.92)$ & $3,016(31.16)$ & $<0.01$ & $0.88 \pm 1.00$ & $0.54 \pm 1.37$ & $0.59 \pm 1.26$ & 0.06 \\
\hline Chest computed tomography & $1,136(8.51)$ & $74(2.03)$ & $177(1.82)$ & 0.42 & $0.38 \pm 0.34$ & $0.03 \pm 0.28$ & $0.03 \pm 0.23$ & 0.07 \\
\hline Pulmonary function test & $12,698(95.14)$ & $2,438(67.03)$ & $4,943(50.91)$ & $<0.01$ & $1.14 \pm 1.09$ & $1.24 \pm 1.56$ & $0.91 \pm 1.35$ & $<0.01$ \\
\hline $\begin{array}{l}\text { ER visit or GW hospitalization } \\
\text { in } 2011\end{array}$ & - & $1,843(50.67)$ & $5,166(53.21)$ & 0.01 & - & $1.42 \pm 2.44$ & $1.44 \pm 2.31$ & 0.61 \\
\hline
\end{tabular}

${ }^{a}$, the entire group of high grade TDL patients (2011-2015); ${ }^{b}$, groups divided by treatment compliance (2012); ${ }^{c}$, for adherent group and non-adherent group; ${ }^{d}$, annual frequency (number/year) per patient; TDL, tuberculosis destroyed lung; SD, standard deviation; ER, emergency room; GW, general ward; ICU, intensive care unit.

Table 3 Costs and days of use of high grade TDL related healthcare

\begin{tabular}{|c|c|c|c|c|c|c|c|c|}
\hline $\begin{array}{l}\text { TDL-related } \\
\text { healthcare } \\
\text { utilization }\end{array}$ & \multicolumn{4}{|c|}{ Cost $(U S \$)^{a}$, mean \pm SD } & \multicolumn{4}{|c|}{ Days of service ${ }^{b}$, mean $\pm S D$} \\
\hline Outpatient service & $1,046.1 \pm 1,015.29$ & $1.081 .40 \pm 1,359.46$ & $978.33 \pm 1,067.84$ & $<0.01$ & $43.54 \pm 32.35$ & $43.31 \pm 36.09$ & $45.72 \pm 37.44$ & $<0.01$ \\
\hline $\begin{array}{l}\text { Outpatient } \\
\text { prescription }\end{array}$ & $1,210.52 \pm 786.72$ & $1,619.30 \pm 1,340.80$ & $1,085.34 \pm 771.10$ & $<0.01$ & - & - & - & - \\
\hline Total & $4,140.95 \pm 3,715.01$ & $4,151.77 \pm 4,084.76$ & $3,592.54 \pm 4,229.93$ & $<0.01$ & $56.21 \pm 45.28$ & $52.96 \pm 50.87$ & $56.67 \pm 50.81$ & $<0.01$ \\
\hline
\end{tabular}

${ }^{a}$, annual average cost (US\$/year) per patient; ${ }^{b}$, annual average frequency (days/year) per patient; ${ }^{\circ}$, the entire group of high grade TDL patients (2011-2015); ${ }^{\text {, }}$ groups divided by treatment compliance (2012); ${ }^{e}$, for Adherent group and non-adherent group; TDL, tuberculosis destroyed lung; SD, standard deviation.

significant $(1.16 \pm 2.24$ vs. $1.23 \pm 2.30, \mathrm{P}=0.11)$; this was also the case for ICU stay $(3.79 \%$ vs. $3.37 \%, \mathrm{P}=0.23 ; 0.05 \pm 0.27$ vs. $0.04 \pm 0.23, \mathrm{P}=0.13)$.

In both groups, the most common test performed by patients at least once was PFT during the study period (67.03\% vs. $50.91 \%, \mathrm{P}<0.01)$. However, there was significant difference in the annual per-patient frequency of these tests only in the PFT $(1.24 \pm 1.56$ vs. $0.91 \pm 1.35$, $\mathrm{P}<0.01)$.

\section{Costs and days of use of high grade TDL related healthcare}

Table 3 summarizes the costs and days of use of healthcare services related to high grade TDL (2011-2015). The annual average cost per patient was approximately US\$1,046.14 $\pm 1,1015.29$ for outpatient services, US\$1,210.52 $\$ 786.72$ for outpatient prescriptions and US\$1,884 for inpatient services. The total average annual service cost per patient was US\$4,140.95 $\pm 3,715.01$. The mean number of days per year on which outpatient and inpatient services were used per patient was $43.54 \pm 32.35$ and $12.66 \pm 30.12$, respectively. The total number of days on which healthcare services was used annually per patient was $56.21 \pm 45.28$.

Table 3 also shows the costs and days of healthcare service utilization for both groups during the study period (2012). The total annual medical cost per patient was higher in the adherent than in the non-adherent 
Table 4 Frequencies and costs of respiratory medications prescribed for patients with high grade TDL

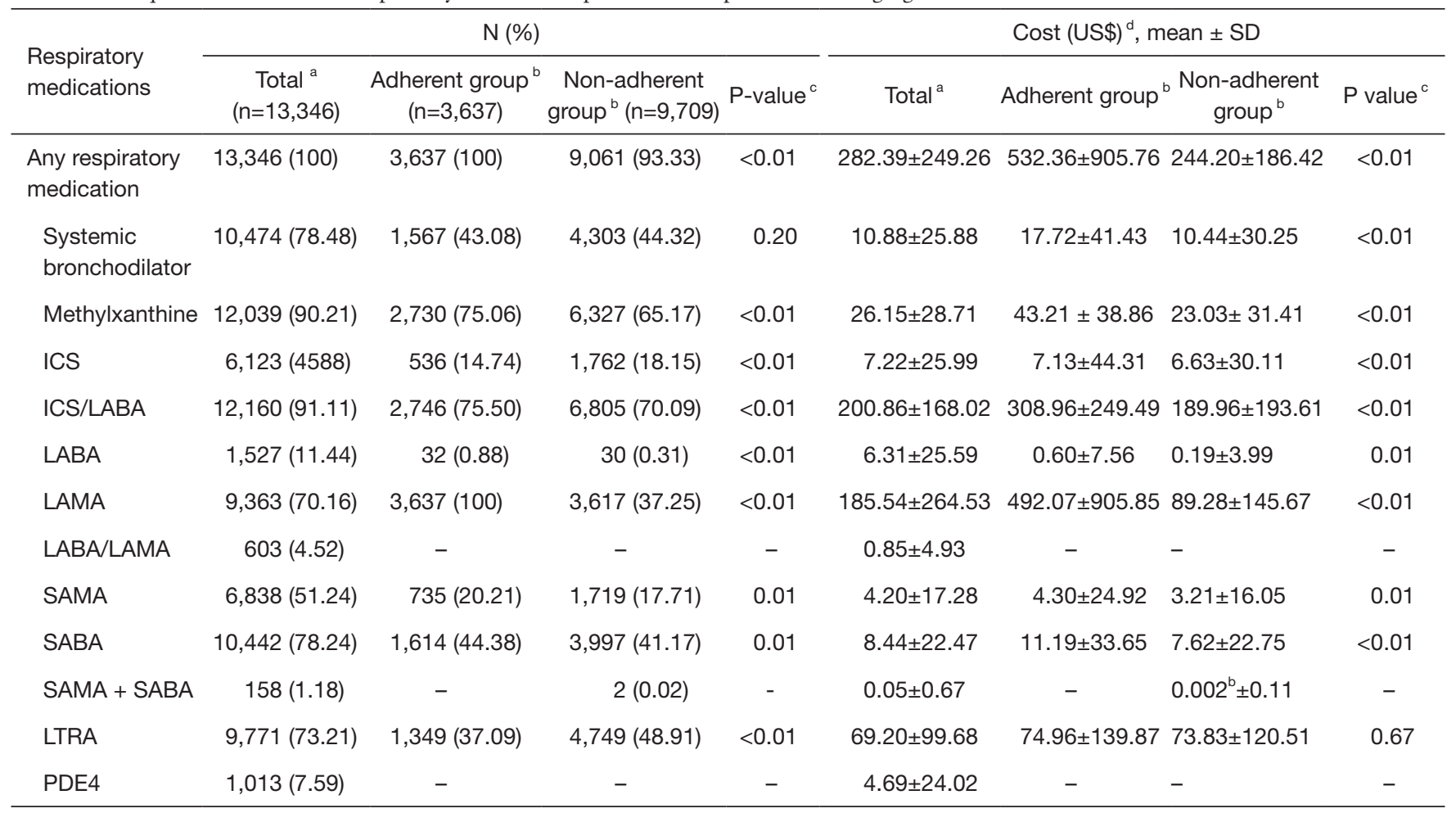

${ }^{a}$, the entire group of high grade TDL patients (2011-2015); ${ }^{b}$, groups divided by treatment compliance (2012); ${ }^{c}$, for adherent group and non-adherent group; ' , annual average cost (US\$/year) per patient. TDL, tuberculosis destroyed lung; SD, standard deviations; ICS, inhaled corticosteroid; ICS/LABA, inhaled corticosteroid/long-acting $\beta 2$ agonist inhaler; LABA, long-acting $\beta 2$ agonist inhaler; LAMA, long-acting muscarinic antagonist inhaler; SAMA, short-acting muscarinic antagonist inhaler; SABA, short-acting $\beta 2$ agonist inhaler; LTRA, leukotriene receptor antagonist; PDE4, phosphodiesterase E4.

group (US $\$ 4,151 \pm 4,084.76$ vs. US $\$ 3,592.54 \pm 4,229.93$, $\mathrm{P}<0.01)$, as was the annual personal cost for outpatient services and prescriptions (US $\$ 1,081.40 \pm 1,359.46 v s$. US\$978.33 $\pm 1,067.84, \mathrm{P}<0.01$ and US $\$ 1,619.30 \pm 1,340.80$ vs. US $\$ 1,085.34 \pm 771.10, \mathrm{P}<0.01)$. By contrast, the costs of inpatients service use were not significantly different (US\$1,451.06 $\pm 3,403.81 v s$. US $\$ 1,528.66 \pm 3,813.21, \mathrm{P}=0.26$ ). The per-patient total number of days was lower in the adherent than in the non-adherent group $(52.96 \pm 50.87 \mathrm{vs}$. $56.67 \pm 50.81, \mathrm{P}<0.01)$. This was the case for both outpatient and inpatient services $(43.31 \pm 36.09$ vs. $45.72 \pm 37.44, \mathrm{P}<0.01$ and $9.65 \pm 33.53$ vs. $10.94 \pm 32.64, \mathrm{P}<0.05)$.

\section{Frequencies and costs of respiratory medications prescribed for high grade TDL patients}

Table 4 shows the frequencies and costs of the medications prescribed during the study period (2011-2015). The annual average cost per patient US\$282.39 \pm 249.26 and the medications most commonly prescribed during the
5 -year study period were ICS/LABAs (91.11\%) and methylxanthines $(90.21 \%)$. ICS/LABAs were the most commonly used inhalers, followed by SABAs (78.24\%), LAMAs $(70.16 \%)$ and short-acting muscarinic antagonist inhalers (SAMAs, 51.24\%). LABA/LAMA and SABA/SAMA combinations were used less frequently. The highest annual cost per person was for ICS/LABAs (US\$200.86 \pm 168.02 ) and LAMAs (US\$185.54 \pm 264.53 ).

The frequencies and costs of respiratory medications used by both groups during the study period are also summarized in Table 4 (2012). All patient in the adherent group used a respiratory medication (100\%) compared to $93.33 \%$ of the patients in the non-adherent group. Only systemic bronchodilators (including oral $\beta 2$ agonists and patches) were used at similar rates by patients in both groups ( $43.08 \%$ vs. $44.32 \%, \mathrm{P}=0.20$ ); the usage patterns (frequencies) of all other medications differed significantly. LAMAs (100\%) were the most commonly used inhalers in the adherent group, followed by ICS/LABAs (75.50\%). 
Table 5 Frequencies of exacerbation in the patients in each group (2012)

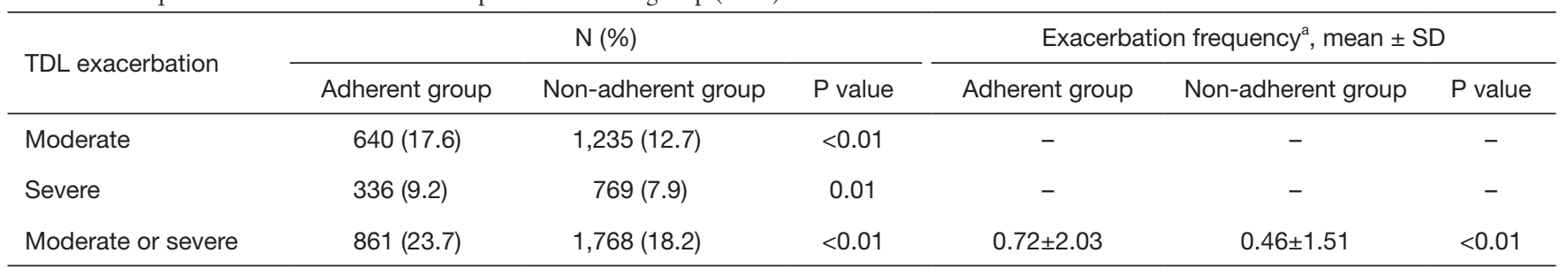

${ }^{\mathrm{a}}$, annual average frequency (number/year) per patient; TDL, tuberculosis destroyed lung; SD, standard deviation.

Table 6 Comparison of exacerbation incidence according to patient group (2012)

\begin{tabular}{|c|c|c|c|}
\hline Exacerbation incidence & $\mathrm{IRR}$ & $95 \% \mathrm{Cl}$ & $P$ value \\
\hline Adjusted IRR ${ }^{\mathrm{a}, \mathrm{b}}$ & 0.64 & $0.57,0.71$ & $<0.01$ \\
\hline
\end{tabular}

${ }^{a}$, reference: adherent group; ${ }^{b}$, adjusted by age, sex, ER visit or hospitalization in previous year (2011, detailed in Table 6), insurance type. $\mathrm{IRR}$, incidence rate ratio; $\mathrm{Cl}$, confidence interval; ER, emergency room.

In comparison, for the non-adherent group, ICS/LABAs $(70.09 \%)$ were the most commonly used inhalers, and SABAs $(41.17 \%)$ were in the second. In addition, inhaler use was more frequent in the adherent than in the nonadherent group (all $\mathrm{P}<0.01$ ). Regarding oral medications, methylxanthines were the most frequently used, especially by the adherent group $(75.06 \%$ vs. $65.17 \%, \mathrm{P}<0.01)$, whereas LTRAs were prescribed significantly more often in the non-adherent group (37.09\% vs. $48.91 \%, \mathrm{P}<0.01)$.

The annual average per-patient cost of medications in the adherent group was approximately US\$532.36 \pm 905.76 , mostly attributable to LAMAs (US\$492.07 $\$ 905.85$ per patient). The cost of ICS/LABAs during the study period was US $\$ 308.96 \pm 249.49$ per patient. In the nonadherent group, the annual average per-patient cost of medications was US $\$ 244.20 \pm 186.42$, mostly accounted for by ICS/LABAs (US\$189.96 \pm 193.61 per patient), followed by LAMAs (US\$89.28 \pm 145.67 per patient). Among oral medications, LTRA costs were the highest in both the adherent (US\$74.96 \pm 139.87 ) and non-adherent (US\$73.83 \pm 120.51 ) groups. With the exception of LTRAs $(\mathrm{P}=0.67)$, all medications costs were higher in the adherent group than in the non-adherent group (all $\mathrm{P}<0.05)$.

\section{Exacerbations of TDL in the two patient groups}

Table 5 shows the frequencies of moderate and severe exacerbations in the two groups (2012). Patients in the adherent group tended to suffer from exacerbations more frequently than did patients in the non-adherent group, whether moderate $(17.60 \%$ vs. $12.72 \%, \mathrm{P}<0.01)$ or severe (9.24 vs. $7.92 \%, \mathrm{P}=0.01$ ). Both the number of patients who suffered from any type of exacerbation at least once and the annual average frequency of exacerbations were higher in the adherent group than in the non-adherent group $23.67 \%$ vs. $18.21 \%$ and $0.72 \pm 2.03$ vs. $0.46 \pm 1.51$, respectively; both $\mathrm{P}<0.01)$.

The difference in incidence of exacerbations was evaluated based of the incident rate ratio (IRR), as shown in Table 6. Adjustments were made for age, sex, number of ER visits or hospitalizations in the previous year (2011) and insurance type. In the adherent group, the crude IRR for exacerbations was 0.64 [95\% confidence interval (CI): $0.58-0.72, \mathrm{P}<0.01]$ and the adjusted IRR was 0.64 (95\% CI: $0.57-0.71, \mathrm{P}<0.01)$.

\section{Factors affecting bealthcare utilization}

Multiple linear regression was performed to identify the factors affecting healthcare utilization (Table 7). Age, sex, adherence, number of ER visits or hospitalizations in the previous year (2011) and insurance type were found to have significantly affected medical costs in 2012. Regarding the number of days of healthcare utilization in 2012, all of the variables except sex $(-1.30, \mathrm{P}=0.13)$ had a significant impact. Non-adherence was negatively related to medical costs and positively related to the number of days on which healthcare services were used. 
Table 7 Multiple linear regression analysis of factors affecting healthcare utilization (2012)

\begin{tabular}{|c|c|c|c|c|c|c|c|c|}
\hline \multirow{2}{*}{ Variables } & \multicolumn{4}{|c|}{ Medical cost (US\$) ${ }^{a}$} & \multicolumn{4}{|c|}{ Days used } \\
\hline & $\beta$ & $P$ value & $\beta$ & $P$ value & $\beta$ & P-value & $\beta$ & $P$ value \\
\hline Age (year) & 46.61 & $<0.01$ & 37.64 & $<0.01$ & 0.824 & $<0.01$ & 0.765 & $<0.01$ \\
\hline Sex (male) & 657.46 & $<0.01$ & 274.87 & $<0.01$ & 2.412 & 0.01 & -1.302 & 0.13 \\
\hline $\begin{array}{l}\text { ER visit or GW hospitalization in } \\
\text { previous year (2011) }\end{array}$ & 696.04 & $<0.01$ & 637.42 & $<0.01$ & 8.668 & $<0.01$ & 7.925 & $<0.01$ \\
\hline Insurance type (medical aid) & $2,042.80$ & $<0.01$ & $1,364.28$ & $<0.01$ & 23.221 & $<0.01$ & 16.092 & $<0.01$ \\
\hline
\end{tabular}

${ }^{\mathrm{a}}$, cost (us\$) per patient; ${ }^{\mathrm{b}}$, per patient; ${ }^{\mathrm{c}}$, reference: adherent group. ER, emergency room; GW, general ward.

\section{Discussion}

The severity and socioeconomic burden of TDL have been expected to be high, however, there has been no extensive investigation. This study is the first to evaluate the clinical characteristics and burden of high grade TDL and to estimate the socioeconomic impact of compliance to inhaler. In particular, using HIRA database enabled nationwide research implementation.

Among the causes of death worldwide, respiratory diseases account for four of the top ten, with COPD ranking third and TB ranking tenth (19). Specifically, TDL and COPD share certain features and both impose a considerable health burden (20). TDL causes air flow obstruction (AFO) $(2,5,13,21,22)$ and has features similar to COPD (23-25). The annual number of hospital admissions among patients with high grade TDL was $1.38 \pm 2.03$ $(0.32 \pm 0.29$ for ICU admissions). The most prevalent comorbidity was cardiovascular diseases, including IHD (22.10\%), CHF (23.48\%), and hypertension (68.85\%). The number of admissions is comparable to COPD and prevalence of comorbidity is similar $(18,26)$.

Medical costs of inpatient services accounted for $83.50 \%$ of outpatients. However, given the fewer medical utilization days of inpatient $(22.52 \%$ of all days) versus outpatient service utilization, medical costs were largely associated with inpatient services, as also reported from a COPD cohort $(18,19,27,28)$. Similar results were seen in intergroup (adherent $v s$. non-adherent group) comparisons. Overall costs were higher in the adherent than in the non-adherent group, which might simply due to high compliance. However, cost for inpatient were similar between two groups $(35.95 \%$ vs. $42.55 \%)$.
Although there are no definitive treatment guidelines for TDL $(2,16,17)$, we found many TDL patients were prescribed COPD medication. In particular, ICS/LABA (91.11\%) was prescribed more frequently than oral drugs and was also the largest proportion in cost. This can be inferred from the fact that TDL and COPD cause similar AFOs as mentioned above (23-25). Rhee et al. showed that LAMA or ICS/LABA can increase FEV1 in TDL patients (2). Yum et al. showed the tiotropium had therapeutic effect on TDL patients (16). More recently, in the multi-center, double-blind clinical trial, indacaterol significantly increased FEV1 and improved dyspnea compared with placebo (17).

In a previous study, $76.8 \%$ (457/595) of TDL patients had AFO with an average of 0.4 exacerbations per year; this rate was similar to that of COPD patients $(0.4 \pm 0.05$ vs. $0.32 \pm 0.03, \mathrm{P}=0.06$ ) (2). In our study, there were large differences in exacerbation frequency between the two groups. Patients in the adherent group tended to have more exacerbations per year $(0.72 \pm 2.03$ vs. $0.46 \pm 1.51$, $\mathrm{P}<0.01)$. However, in real world study, compliance may reflect disease severity. Patients with less symptom with less exacerbation usually used inhaler less. Thus, the more frequent exacerbations seen in the adherent group in this study may be attributable to the patient selection criteria. We divided the high grade TDL population according to compliance based only on the ICD and billing codes entered into the HIRA system, but we could not determine disease severity. Nevertheless, even taking this shortcoming into consideration, there were fewer days of healthcare service utilization (both outpatient and inpatient) in the adherent group. Adherence also significantly reduced the number of days of healthcare service use, as shown in the in multiple linear regression analysis. 
Our study had several limitations. PFT and disease symptoms are among the most important factors affecting the outcomes of patients with chronic AFO. However, PFT or symptom data are not contained within the HIRA database. Consequently, our adjustment for disease severity may not have been sufficient and it is possible that patients with severe symptoms or poor PFT results were more likely to be included in the adherent group. Because the HIRA database is a claims database, it contains relatively few measurable variables relevant to healthcare burden; only age, sex, treatment compliance, insurance type and hospital visits in the previous year could be analyzed. Second, we only analyzed one-year outcome data. Third, we did not test the normality of the data.

Despite these limitations, this was the first nationwide study to evaluate high grade TDL and estimate the socioeconomic burden of the disease. Issues such as medical service utilization, costs and drug usage status were analyzed accurately using officially certified HIRA claims data. Our results provide evidence of the positive effect of adherence and inhaled bronchodilator use on certain outcomes, especially days of healthcare utilization, in patients with high grade TDL.

\section{Conclusions}

High grade TDL imposes a considerable socioeconomic burden. Patients who adhered to treatment regimens had higher medical costs than did not. More exacerbation occurred in adherence group. Nonetheless, adhrerence was significantly associated with less healthcare utilization. Efforts to prevent hospitalization due to disease exacerbation or comorbidities and to delay the progression of TDL itself, are important and should be taken to alleviate the socioeconomic burden associated with high grade TDL. Prospective clinical trial for TDL patients is needed to evaluate the effect of inhaled bronchodilator.

\section{Acknowledgments}

Funding: This study was supported by a grant from the Ministry of Health and Welfare, Republic of Korea (grant no. HC16C-2254-010017).

\section{Footnote}

Conflicts of Interest: The authors have no conflicts of interest to declare.
Ethical Statement: The authors are accountable for all aspects of the work in ensuring that questions related to the accuracy or integrity of any part of the work are appropriately investigated and resolved. To protect their confidentiality, all patients were assigned and anonymous identification code in the HIRA database that prevented the authors from identifying them. The study was approved by the Institutional Review Board of Seoul St. Mary's Hospital (No. KC18ZESE0458), which waived the requirement for informed consent.

\section{References}

1. WHO global tuberculosis report 2017.

2. Rhee CK, Yoo KH, Lee JH, et al. Clinical characteristics of patients with tuberculosis-destroyed lung. Int J Tuberc Lung Dis 2013;17:67-75.

3. Ryu YJ, Lee JH, Chun EM, et al. Clinical outcomes and prognostic factors in patients with tuberculous destroyed lung. Int J Tuberc Lung Dis 2011;15:246-50.

4. Kim SJ, Lee J, Park YS, et al. Effect of airflow limitation on acute exacerbations in patients with destroyed lungs by tuberculosis. J Korean Med Sci 2015;30:737-42.

5. Lee JH, Chang JH. Lung function in patients with chronic airflow obstruction due to tuberculous destroyed lung. Respir Med 2003;97:1237-42.

6. Kim WY, Kim MH, Jo EJ, et al. Predicting Mortality in Patients with Tuberculous Destroyed Lung Receiving Mechanical Ventilation. Tuberc Respir Dis (Seoul) 2018;81:247-55.

7. Allwood BW, Gillespie R, Galperin-Aizenberg M, et al. Obstructive pulmonary disease in patients with previous tuberculosis: Pathophysiology of a community-based cohort. S Afr Med J 2017;107:440-5.

8. Allwood BW, Rigby J, Griffith-Richards S, et al. Histologically confirmed tuberculosis-associated obstructive pulmonary disease. Int J Tuberc Lung Dis 2019;23:552-4.

9. Byrne AL, Marais BJ, Mitnick CD, et al. Tuberculosis and chronic respiratory disease: a systematic review. Int J Infect Dis 2015;32:138-46.

10. Byrne AL, Marais BJ, Mitnick CD, et al. Risk factors for and origins of COPD. Lancet 2015;385:1723-4.

11. Hwang YI, Kim JH, Lee CY, et al. The association between airflow obstruction and radiologic change by tuberculosis. J Thorac Dis 2014;6:471-6.

12. Jordan TS, Spencer EM, Davies P. Tuberculosis, bronchiectasis and chronic airflow obstruction. 
Respirology 2010;15:623-8.

13. Menezes AM, Hallal PC, Perez-Padilla R, et al. Tuberculosis and airflow obstruction: evidence from the PLATINO study in Latin America. Eur Respir J 2007;30:1180-5.

14. Myong JP, Yoon HK, Rhee CK, et al. Risk factors for lung function impairment among the general nonsmoking Korean population. Int J Tuberc Lung Dis 2015;19:1019-26.

15. Rhee CK. High prevalence of chronic obstructive pulmonary disease in Korea. Korean J Intern Med 2016;31:651-2.

16. Yum HK, Park IN. Effect of inhaled tiotropium on spirometric parameters in patients with tuberculous destroyed lung. Tuberc Respir Dis (Seoul) 2014;77:167-71.

17. Kim CJ, Yoon HK, Park MJ, et al. Inhaled indacaterol for the treatment of COPD patients with destroyed lung by tuberculosis and moderate-to-severe airflow limitation: results from the randomized INFINITY study. Int J Chron Obstruct Pulmon Dis 2017;12:1589-96.

18. Kim J, Rhee CK, Yoo KH, et al. The health care burden of high grade chronic obstructive pulmonary disease in Korea: analysis of the Korean Health Insurance Review and Assessment Service data. Int J Chron Obstruct Pulmon Dis 2013;8:561-8.

19. Lopez-Campos JL, Tan W, Soriano JB. Global burden of COPD. Respirology 2016;21:14-23.

20. Chakrabarti B, Calverley PM, Davies PD. Tuberculosis and its incidence, special nature, and relationship with

Cite this article as: Han D, Lee HY, Kim K, Kim T, Oh YM, Rhee CK. Burden and clinical characteristics of high grade tuberculosis destroyed lung: a nationwide study. J Thorac Dis 2019;11(10):4224-4233. doi: 10.21037/jtd.2019.09.63 chronic obstructive pulmonary disease. Int J Chron Obstruct Pulmon Dis 2007;2:263-72.

21. Vestbo J, Edwards LD, Scanlon PD, et al. Changes in forced expiratory volume in 1 second over time in COPD. N Engl J Med 2011;365:1184-92.

22. Willcox PA, Ferguson AD. Chronic obstructive airways disease following treated pulmonary tuberculosis. Respir Med 1989;83:195-8.

23. Hnizdo E, Singh T, Churchyard G. Chronic pulmonary function impairment caused by initial and recurrent pulmonary tuberculosis following treatment. Thorax 2000;55:32-8.

24. Pasipanodya JG, Miller TL, Vecino M, et al. Pulmonary impairment after tuberculosis. Chest 2007;131:1817-24.

25. Lee SW, Kim YS, Kim DS, et al. The risk of obstructive lung disease by previous pulmonary tuberculosis in a country with intermediate burden of tuberculosis. J Korean Med Sci 2011;26:268-73.

26. Kim C, Yoo KH, Rhee CK, et al. Health care use and economic burden of patients with diagnosed chronic obstructive pulmonary disease in Korea. Int J Tuberc Lung Dis 2014;18:737-43.

27. Yoo KH, Ahn HR, Park JK, et al. Burden of Respiratory Disease in Korea: An Observational Study on Allergic Rhinitis, Asthma, COPD, and Rhinosinusitis. Allergy Asthma Immunol Res 2016;8:527-34.

28. Anzueto A, Sethi S, Martinez FJ. Exacerbations of chronic obstructive pulmonary disease. Proc Am Thorac Soc 2007;4:554-64. 